\title{
Composite Antidisturbance Control for a Class of Nonlinear Stochastic Systems via Disturbance Observer
}

\author{
Yunlong Liu, ${ }^{1}$ Yumin Zhang, ${ }^{1}$ and Xiuming Yao ${ }^{2}$ \\ ${ }^{1}$ School of Instrumentation Science and Opto-Electronics Engineering, Beihang University, Beijing 100191, China \\ ${ }^{2}$ Hebei Engineering Research Center of Simulation and Optimized Control for Power Generation, \\ North China Electric Power University, Baoding 071003, China
}

Correspondence should be addressed to Yunlong Liu; liuyunlongbuaa@163.com

Received 8 October 2013; Accepted 17 November 2013

Academic Editor: Xiaojie Su

Copyright (C) 2013 Yunlong Liu et al. This is an open access article distributed under the Creative Commons Attribution License, which permits unrestricted use, distribution, and reproduction in any medium, provided the original work is properly cited.

\begin{abstract}
The stabilization problem is investigated in this paper for a class of nonlinear systems with disturbances. The disturbances are supposed to be classified into two types. One type in the input channel is generated by an exogenous system, which can represent the constant or harmonic signals with unknown phase and magnitude. The other type is stochastic disturbance. Two kinds of nonlinear dynamics in the plants are considered, respectively, which correspond to the known and unknown functions. By integrating the disturbance observers with conventional control method, the first type of disturbances can be estimated and rejected. Simultaneously, the desired dynamic performances can be guaranteed. An example is given to show the effectiveness of the proposed scheme.
\end{abstract}

\section{Introduction}

Though the stochastic stabilization theory emerged in the 1960s [1], the progress has been slow. This was mainly due to a fundamental theory obstacle in the Lyapunov analysis; the Itô differentiation introduces not only the gradient but also the Hessian term of the Lyapunov function. Along with the advances in differential geometric theory [2] and the discovery of a simple constructive formula for Lyapunov stabilization [3], the stochastic stabilization problem was reexamined and some constructive results have been achieved. The stabilization of nonlinear stochastic systems was considered in the work of Florchinger [4-7], who, among other things, extended the concept of control Lyapunov functions and Sontag's stabilization formula to stochastic setting. Pan and Başer [8] solved the stabilization problem for a class of strict-feedback systems representative of stabilization results for deterministic systems. Deng and Krstić [9] developed a simpler control strategy for strict-feedback systems and then extended the results on inverse optimal stabilization for general systems to the stochastic case. The authors of [10] considered the dissipativity analysis and dissipativity-based sliding mode control for a class of continuous-time switched stochastic systems and [11] designed multistep predictive controller for a class of Markov jump convex polyhedron linear parameter time-varying systems with both constraints on input and output. The adaptive neural tracking control problem was the concern in [12] for a class of strict-feedback stochastic nonlinear systems with unknown dead zone. However, most of these results were focused on systems that only have one kind of disturbance-stochastic disturbance. In [13], Hinrichsen proposed the stochastic robust control method for systems with deterministic and stochastic disturbances, which enabled us to deal with a broader class of systems. However, only stability of the nominal system in the absence of deterministic disturbances was the concern in this approach, which means that the stability cannot be guaranteed in the presence of both deterministic and stochastic disturbances.

Disturbance-observer-based control (DOBC) approach, which is based on the idea of feed-forward compensation, appeared in the late 1980s and has attracted considerable attention in control theory literatures [14-17]. The controller design of this method can be accomplished in two steps. First, a disturbance observer is designed to estimate the deterministic disturbance and then compensate it. Second, feedback controller is designed to stabilize the nominal system without disturbance. DOBC approach has its roots in 
many mechanical applications in the last two decades, in particular for linear systems [18-20]. Recently, some attempts have been made to establish theoretic justification of these DOBC applications and extend DOBC from linear systems to nonlinear systems [21, 22]. Besides, [23] designed output feedback controller for a class of Markovian jump repeated scalar nonlinear systems and [24] investigated the problem of composite DOBC and $H_{\infty}$ control for Markovian jump systems with nonlinearity and multiple disturbances.

This paper considers the application of DOBC approach to a class of nonlinear stochastic systems. The nonlinear dynamics are described by known and unknown nonlinear functions, respectively. And apart from the stochastic noises, the deterministic disturbance is supposed to be generated by an exogenous system as investigated in $[18,19]$, which is not confined to be bounded in norm [25]. By using the disturbance estimation, the DOBC strategy can be integrated with the conventional stabilization controllers to reject the deterministic disturbance and globally stabilize the closed-loop systems in probability. Finally, simulations on an A4D aircraft model show the effectiveness of the proposed approaches.

\section{Problem Statement}

The following MIMO stochastic system with nonlinearity is described as

$$
\begin{aligned}
d x(t)= & \left\{A x(t)+F_{0} f(x(t), t)+B[u(t)+v(t)]\right\} d t \\
& +A_{0} x(t) d \omega
\end{aligned}
$$

where $x(t) \in R^{n}, u(t) \in R^{m}$ and $v(t) \in R^{m}$, are the state, control input, and disturbance, respectively. $f(x(t), t)$ is a nonlinear vector function satisfying bounded condition as described in Assumption 1. $\omega$ is an $r$-dimensional standard Brownian motion defined on a complete probability space $(\Omega, \mathscr{F}, \mathscr{P})$ with $\Omega$ being a sample space, $\mathscr{F}$ being a $\sigma$-field, and $\mathscr{P}$ being the probability measure. $A, B, F_{0}$, and $A_{0}$ are given system matrices with corresponding dimensions.

Assumption 1. For any $x_{j}(t) \in R^{n}, j=1,2$, nonlinear function $f(x(t), t)$ satisfy

$$
\begin{gathered}
f(0, t)=0, \\
\left\|f\left(x_{1}(t), t\right)-f\left(x_{2}(t), t\right)\right\| \leq\left\|U\left(x_{1}(t)-x_{2}(t)\right)\right\|,
\end{gathered}
$$

where $U$ is a given constant weighting matrix.

Assumption 2. The disturbance $v(t)$ in the control input path is supposed to be generated by the following exogenous systems:

$$
\dot{\omega}(t)=W \omega(t), \quad v(t)=V \omega(t),
$$

where $W \in R^{r \times r}, V \in R^{m \times r}$ are known constant weighting matrices.

Remark 3. In fact, many kinds of disturbances in engineering can be described by Assumption 2, for example, unknown constant and harmonics with unknown phase and magnitude.
The following assumption is the necessary condition for the DOBC problem.

Assumption 4. $(A, B)$ is controllable and $(W, B V)$ is observable.

In this paper, we suppose system states are available, which means that only the estimation of disturbance needs to be focused on. In this situation, the objective of DOBC is to design an observer for system (1) to estimate the unknown disturbance $v(t)$, and then construct a composite controller with the disturbance estimation and a conventional controller so that the disturbance can be rejected and the stability in probability of the resulting composite system can be guaranteed.

\section{DOBC for the Case with Known Nonlinearity}

In this section, the nonlinearity function $f(x(t), t)$ is supposed to be given and Assumptions 1, 2, and 4 hold. Due to the fact that the states of system are available, the disturbance observer is designed as

$$
\widehat{v}(t)=V \widehat{\widehat{\omega}}(t), \quad \widehat{\omega}(t)=s(t)-L x(t),
$$

where $\widehat{\omega}(t)$ is the estimation of $\omega(t)$ and $s(t)$ is the auxiliary variable generated by

$$
\begin{aligned}
d s(t)=[ & (W+L B V)(s(t)-L x(t)) \\
& \left.+L\left(A x(t)+F_{0} f(x, t)+B u(t)\right)\right] d t .
\end{aligned}
$$

Denote the estimation error as $e_{\varrho}(t)=\omega(t)-\widehat{\omega}(t)$. Based on (1), (3), (4), and (5), the error dynamics satisfy

$$
d e_{\omega}(t)=(W+L B V) e_{\omega} d t+L A_{0} x(t) d \omega .
$$

The objective of disturbance rejection can be achieved by designing the observer gain $L$ such that (6) satisfies the desired stability in probability.

For DOBC strategy, the controller is usually selected as $[22,26-28]$

$$
u(t)=-\widehat{v}(t)+K x(t)
$$

where $\widehat{v}(t)$ is to compensate the disturbance in control input path and $K$ is the conventional feedback gain needed to be determined later.

By substituting (7) into (1), the closed loop system can be written in the following form:

$$
\begin{aligned}
d x(t)= & \left\{(A+B K) x(t)+F_{0} f(x(t), t)+B V e_{\emptyset}(t)\right\} d t \\
& +A_{0} x(t) d \omega .
\end{aligned}
$$

Combining (8) with (6), the composite system is described as

$$
d \bar{x}(t)=\{\bar{A} \bar{x}(t)+F f(x, t)\} d t+\bar{A}_{0} \bar{x}(t) d \omega
$$


where

$$
\begin{gathered}
\bar{x}(t)=\left[\begin{array}{c}
x(t) \\
e_{\omega}(t)
\end{array}\right], \quad \bar{A}=\left[\begin{array}{cc}
A+B K & B V \\
0 & W+L B V
\end{array}\right], \\
F=\left[\begin{array}{c}
F_{0} \\
0
\end{array}\right], \quad \bar{A}_{0}=\left[\begin{array}{cc}
A_{0} & 0 \\
L A_{0} & 0
\end{array}\right] .
\end{gathered}
$$

In the following, the objective is to design gain $L$ and $K$ such that system (9) is asymptotical stabilization in probability. For the convenience of research, the following lemma is presented.

Lemma 5 (see $[29,30])$. For a given stochastic system

$$
d x(t)=g(x, t) d t+h(x, t) d \omega, \quad x\left(t_{0}\right)=x_{0},
$$

if there exists function $V(x, t) \in C^{1,2}, \mu_{1}(\cdot), \mu_{2}(\cdot) \in \mathscr{K}_{\infty}$, constants $c_{1}>0$, and a nonnegative $M(x, t)$, such that

$$
\mu_{1}(x) \leq V(x, t) \leq \mu_{2}(x), \quad \mathscr{L} V \leq-c_{1} M(x, t),
$$

then, one has the following.

(i) The equilibrium $x=0$ is globally stable in probability and the solution $x(t)$ satisfied $P\left\{\lim _{t \rightarrow \infty} M(x(t))=\right.$ $0\}=1$, when $g(0, t)=0, h(0, t)=0$, and $M(x, t)=$ $M(x)$ is continuous.

(ii) The equilibrium $x=0$ is globally asymptotically stable in probability, when $g(0, t)=0, h(0, t)=0$, and $M(x, t)$ is positive definite.

Here, the differential operator $\mathscr{L}$ for differentiable function $V(x, t)$ is defined as

$$
\mathscr{L} V=\frac{\partial V}{\partial x} g(x, t)+\frac{1}{2} \operatorname{Tr}\left\{h(x, t)^{T} \frac{\partial^{2} V}{\partial x^{2}} h(x, t)\right\} .
$$

Theorem 6. Consider system (1) with disturbance (3) under Assumptions 1, 2, and 4. For some $\lambda>0$, if there exist $P_{1}=$ $P_{1}^{T}>0, R_{1}$ and constant $\beta>0$ satisfying

$$
\left[\begin{array}{cc}
\operatorname{sym}\left(P_{1} W+R_{1} B V\right) & V^{T} B^{T} \\
B V & -\beta^{2} I
\end{array}\right]<0
$$

and $Q_{2}=Q_{2}^{T}>0, R_{2}$ satisfying

$$
\left[\begin{array}{cccc}
\Xi & Q_{2} A_{0}^{T} & Q_{2} A_{0}^{T} R_{1}^{T} & Q_{2} U^{T} \\
A_{0} Q_{2} & -Q_{2} & 0 & 0 \\
R_{1} A_{0} Q_{2} & 0 & -P_{1} & 0 \\
U Q_{2} & 0 & 0 & -\lambda^{2} I
\end{array}\right]<0,
$$

where $\Xi=\operatorname{sym}\left(A Q_{2}+B R_{2}\right)+\lambda^{2} F_{0} F_{0}^{T}+\beta^{2} I$, then the closed loop system (8) under DOBC law (7) with gain $K=R_{2} Q_{2}^{-1}$ and observer (4) with gain $L=P_{1}^{-1} R_{1}$ are global asymptotical stabilization in probability.

Proof. Define

$$
\begin{gathered}
\Sigma_{1}\left(e_{\omega}, t\right)=e_{\omega}^{T} P_{1} e_{\omega}, \\
\Sigma_{2}(x, t)=x^{T} P_{2} x+\frac{1}{\lambda^{2}} \int_{0}^{t}\left[\|U x(t)\|^{2}-\|f(x, t)\|^{2}\right] d \tau,
\end{gathered}
$$

where $P_{2}^{-1}=Q_{2}$.
It is noted that for all $x$ and $e_{\omega}, \Sigma_{1} \geq 0, \Sigma_{2} \geq 0$. In addition, along with (6), (8), the Itó differential of $\Sigma_{1}, \Sigma_{2}$ satisfies

$$
\begin{aligned}
\mathscr{L} \Sigma_{1}= & e_{\varpi}^{T}\left[P_{1}(W+L B V)+(W+L B V)^{T} P_{1}\right] e_{\varpi} \\
& +x^{T} A_{0}^{T} L^{T} P_{1} L A_{0} x, \\
\mathscr{L} \Sigma_{2}= & x^{T}\left[P_{2}(A+B K)+(A+B K)^{T} P_{2}\right] x \\
& +2 x^{T} P_{2} F_{0} f+2 x^{T} P_{2} B V e_{\emptyset}+\frac{1}{\lambda^{2}} x^{T} U^{T} U x \\
& +x^{T} A_{0}^{T} P_{2} A_{0} x-\frac{1}{\lambda^{2}} f^{T} f .
\end{aligned}
$$

Via Young inequality, we get

$$
\begin{gathered}
2 x^{T} P_{2} F_{0} f \leq \lambda^{2} x^{T} P_{2} F_{0} F_{0}^{T} P_{2} x+\frac{1}{\lambda^{2}} f^{T} f, \\
2 x^{T} P_{2} B V e_{\varpi} \leq \beta^{2} x^{T} P_{2} P_{2} x+\frac{1}{\beta^{2}} e_{\varpi}^{T} V^{T} B^{T} B V e_{\omega} .
\end{gathered}
$$

Then we have

$$
\begin{aligned}
\mathscr{L} \Sigma_{2} \leq x^{T}\{ & \operatorname{sym}\left[P_{2}(A+B K)\right]+A_{0}^{T} P_{2} A_{0}+\frac{1}{\lambda^{2}} U^{T} U \\
& \left.+\lambda^{2} P_{2} F_{0} F_{0}^{T} P_{2}+\beta^{2} P_{2} P_{2}\right\} x+\frac{1}{\beta^{2}} e_{\omega}^{T} V^{T} B^{T} B V e_{\omega} .
\end{aligned}
$$

A Lyapunov function candidate for (9) is chosen as $\Sigma\left(x, e_{\omega}, t\right)=\Sigma_{1}\left(e_{\omega}, t\right)+\Sigma_{2}(x, t)$; hence, it is easy to get

$$
\begin{aligned}
\mathscr{L} \Sigma \leq & e_{Ф}^{T}\left\{\operatorname{sym}\left[P_{1}(W+L B V)\right]+\frac{1}{\beta^{2}} V^{T} B^{T} B V\right\} e_{\varpi} \\
+ & x^{T}\left\{\operatorname{sym}\left[P_{2}(A+B K)\right]+A_{0}^{T} P_{2} A_{0}+\frac{1}{\lambda^{2}} U^{T} U\right. \\
& \left.\quad+\lambda^{2} P_{2} F_{0} F_{0}^{T} P_{2}+\beta^{2} P_{2} P_{2}+A_{0}^{T} L^{T} P_{1} L A_{0}\right\} x \\
= & e_{\varpi}^{T} \Pi_{1} e_{\varpi}+x^{T} \Pi_{2} x,
\end{aligned}
$$

where

$$
\begin{aligned}
\Pi_{1}= & \operatorname{sym}\left[P_{1}(W+L B V)\right]+\frac{1}{\beta^{2}} V^{T} B^{T} B V, \\
\Pi_{2}= & \operatorname{sym}\left[P_{2}(A+B K)\right]+A_{0}^{T} P_{2} A_{0}+\frac{1}{\lambda^{2}} U^{T} U \\
& +\lambda^{2} P_{2} F_{0} F_{0}^{T} P_{2}+\beta^{2} P_{2} P_{2}+A_{0}^{T} L^{T} P_{1} L A_{0} .
\end{aligned}
$$

Using Lemma 5, it can be verified that system (9) is global asymptotical stabilization in probability if $\Pi_{1}<0$ and $\Pi_{2}<0$ hold.

Based on Schur complement, $\Pi_{1}<0$ is equivalent to $\Pi_{10}<0$, where

$$
\Pi_{10}=\left[\begin{array}{cc}
\operatorname{sym}\left[P_{1} W+R_{1} B V\right] & V^{T} B^{T} \\
B V & -\beta^{2} I
\end{array}\right],
$$


and $\Pi_{2}<0$ is equivalent to $\Pi_{21}<0$, where

$$
\Pi_{21}=\left[\begin{array}{cccc}
\Xi_{0} & A_{0}^{T} & A_{0}^{T} L^{T} P_{1} & U^{T} \\
* & -P_{2}^{-1} & 0 & 0 \\
* & * & -P_{1} & 0 \\
* & * & * & -\lambda^{2} I
\end{array}\right]
$$

and $\Xi_{0}=\operatorname{sym}\left[P_{2}(A+B K)\right]+\lambda^{2} P_{2} F_{0} F_{0}^{T} P_{2}+\beta^{2} P_{2} P_{2}$. In addition, * represents the corresponding elements in the symmetric matrix.

$\Pi_{21}$ is premultiplied and postmultiplied simultaneously by $\operatorname{diag}\left\{Q_{2}, I, I, I\right\}$; then it is equivalent to $\Pi_{20}$, where

$$
\Pi_{20}=\left[\begin{array}{cccc}
\Xi & Q_{2} A_{0}^{T} & Q_{2} A_{0}^{T} R_{1}^{T} & Q_{2} U^{T} \\
* & -Q_{2} & 0 & 0 \\
* & * & -P_{1} & 0 \\
* & * & * & -\lambda^{2} I
\end{array}\right]
$$

and $\Xi=\operatorname{sym}\left[(A+B K) Q_{2}\right]+\lambda^{2} F_{0} F_{0}^{T}+\beta^{2} I$.

Thus, (14), (15) can be obtained.

On the other hand, (14), (15) hold, meaning that there exist $\alpha_{1}>0, \alpha_{2}>2$ such that $\Pi_{10}<-\alpha_{1} I, \Pi_{20}<-\alpha_{2} I$; that is, $\Pi_{1}<-\alpha_{1} I, \Pi_{2}<-\alpha_{2} I$. Hence, we have

$$
\begin{aligned}
\mathscr{L} \Sigma & \leq e_{\omega}^{T} \Pi_{1} e_{\omega}+x^{T} \Pi_{2} x \\
& \leq-\alpha_{1}\left\|e_{\omega}\right\|^{2}-\alpha_{2}\|x\|^{2} \\
& \leq-\min \left\{\alpha_{1}, \alpha_{2}\right\}\left(\|x\|^{2}+\left\|e_{\omega}\right\|^{2}\right) \\
& =-\min \left\{\alpha_{1}, \alpha_{2}\right\}\|\bar{x}\|^{2} .
\end{aligned}
$$

Therefore, the closed-loop system (9) is global asymptotical stabilization in probability when the control gain is selected as $K=R_{2} Q_{2}^{-1}$ and observer gain is selected as $L=P_{1}^{-1} R_{1}$. The proof is completed.

\section{DOBC for the Case with Unknown Nonlinearity}

In this section, the nonlinear function $f(x(t), t)$ is supposed to be unknown, which means disturbance observer should be designed different from Section 3. In this case, the disturbance observer can be constructed as

$$
\begin{gathered}
\widehat{v}(t)=V \widehat{\widehat{\omega}}(t), \quad \widehat{\omega}(t)=s(t)-L x(t), \\
d s(t)=(W+L B V)(s(t)-L x(t))+L(A x(t)+B u(t)) d t .
\end{gathered}
$$

Compared with (6), the estimation error $e_{\varrho}(t)=\omega(t)-$ $\widehat{\omega}(t)$ satisfies

$$
d e_{\oplus}(t)=\left[(W+L B V) e_{\varpi}+L F_{0} f(x, t)\right] d t+L A_{0} x(t) d \omega .
$$

Thus, the composite system combined (8) with (27) is given by

$$
d \bar{x}(t)=\{\bar{A} \bar{x}(t)+F f(x, t)\} d t+\bar{A}_{0} \bar{x}(t) d \omega,
$$

where

$$
\begin{gathered}
\bar{x}(t)=\left[\begin{array}{c}
x(t) \\
e_{\omega}(t)
\end{array}\right], \quad \bar{A}=\left[\begin{array}{cc}
A+B K & B V \\
0 & W+L B V
\end{array}\right], \\
F=\left[\begin{array}{c}
F_{0} \\
L F_{0}
\end{array}\right], \quad \bar{A}_{0}=\left[\begin{array}{cc}
A_{0} & 0 \\
L A_{0} & 0
\end{array}\right] .
\end{gathered}
$$

The objective of this section is similar to Section 3, that is, to design gain $L$ and $K$ such that system (28) is asymptotically stable in probability.

Theorem 7. Consider system (1) with disturbance (3) under Assumptions 1, 2, and 4. For some $\lambda_{1}>0, \lambda_{2}>0$, if there exist $P_{1}=P_{1}^{T}>0, R_{1}$ and constant $\beta>0$ satisfying

$$
\left[\begin{array}{ccc}
\operatorname{sym}\left(P_{1} W+R_{1} B V\right) & R_{1} F_{0} & V^{T} B^{T} \\
* & -\lambda_{1}^{-2} I & 0 \\
* & * & -\beta^{2} I
\end{array}\right]<0
$$

and $Q_{2}=Q_{2}^{T}>0, R_{2}$ satisfying

$$
\left[\begin{array}{ccccc}
\bar{\Xi} & Q_{2} A_{0}^{T} & Q_{2} A_{0}^{T} R_{1}^{T} & Q_{2} U^{T} & Q_{2} U^{T} \\
* & -Q_{2} & 0 & 0 & 0 \\
* & * & -P_{1} & 0 & 0 \\
* & * & * & -\lambda_{1}^{2} I & 0 \\
* & * & * & * & -\lambda_{2}^{2} I
\end{array}\right]<0 \text {, }
$$

where $\bar{\Xi}=\operatorname{sym}\left(A Q_{2}+B R_{2}\right)+\lambda_{2}^{2} F_{0} F_{0}^{T}+\beta^{2} I$, then the closed loop system (28) under DOBC law (7) with gain $K=R_{2} Q_{2}^{-1}$ and observer (26) with gain $L=P_{1}^{-1} R_{1}$ is global asymptotical stabilization in probability.

Proof. Let

$$
\begin{aligned}
& \bar{\Sigma}_{1}\left(e_{\omega}, t\right)=e_{\omega}^{T} P_{1} e_{\omega}+\frac{1}{\lambda_{1}^{2}} \int_{0}^{t}\left[\|U x(t)\|^{2}-\|f(x, t)\|^{2}\right] d \tau, \\
& \bar{\Sigma}_{2}(x, t)=x^{T} P_{2} x+\frac{1}{\lambda_{2}^{2}} \int_{0}^{t}\left[\|U x(t)\|^{2}-\|f(x, t)\|^{2}\right] d \tau,
\end{aligned}
$$

where $P_{2}^{-1}=Q_{2}$. And a Lyapunov function candidate for (28) is chosen as $\bar{\Sigma}\left(x, e_{\omega}, t\right)=\bar{\Sigma}_{1}\left(e_{\omega}, t\right)+\bar{\Sigma}_{2}(x, t)$. The following proof procedure can be given similarly to that of the proof for Theorem 6.

\section{Simulation Example}

In [21], DOBC strategy was employed to a deterministic system of A4D aircraft with disturbance, and better system performance was obtained than some previous results [31]. However, stochastic noise should be considered when higher precision of system performance was required. In this section, the stochastic system that represents the longitudinal dynamics of A4D aircraft is considered, which is described as follows:

$$
\begin{aligned}
d x(t)= & \left\{A x(t)+F_{0} f(x, t)+B[u(t)+v(t)]\right\} d t \\
& +A_{0} x(t) d \omega
\end{aligned}
$$




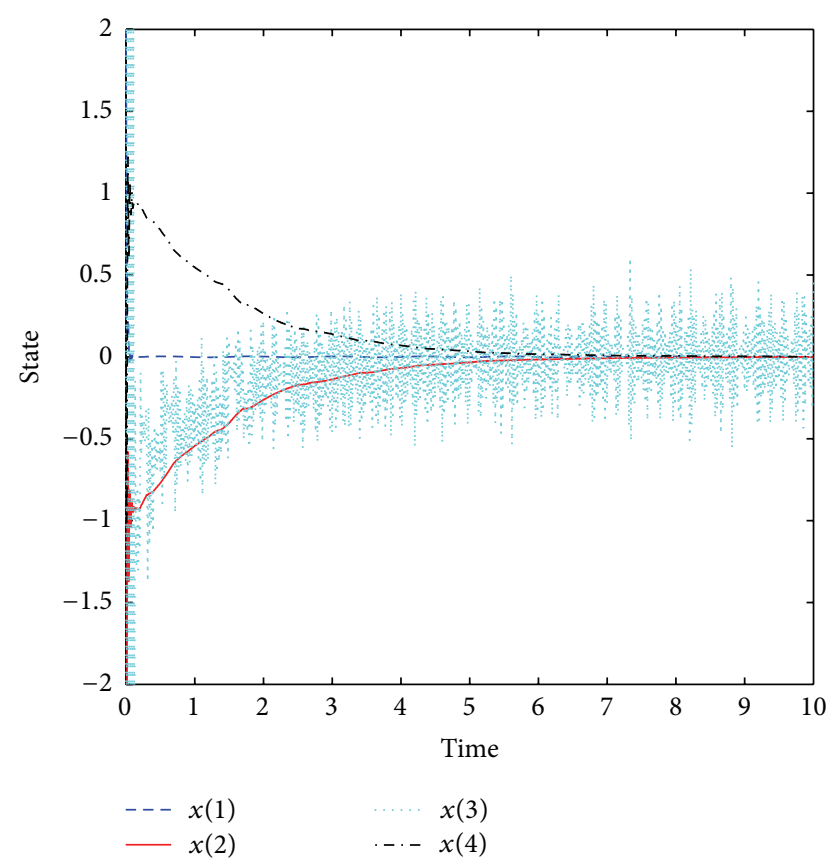

FIGURE 1: System performance using stochastic robust control strategy.

with the following coefficient:

$$
\begin{aligned}
A & =\left[\begin{array}{cccc}
-0.0605 & 32.37 & 0 & 32.2 \\
-0.00014 & -1.475 & 1 & 0 \\
-0.0111 & -34.72 & -2.793 & 0 \\
0 & 0 & 1 & 0
\end{array}\right], \\
F_{0} & =\left[\begin{array}{c}
0 \\
0 \\
0 \\
60
\end{array}\right], \\
A_{0} & =\left[\begin{array}{cccc}
1 & 0 & 0 & 0 \\
0 & 0.3 & 0 & 0.2 \\
0 & 0 & 0 & 0 \\
0 & 0 & 0 & 0.5
\end{array}\right] .
\end{aligned}
$$

The parameter matrices for disturbance $v(t)$ that are described by (3) are given by

$$
W=\left[\begin{array}{cc}
0 & 5 \\
-5 & 0
\end{array}\right], \quad V=\left[\begin{array}{ll}
25 & 10
\end{array}\right]
$$

Case 1 (with known nonlinearity). In this case, the nonlinear dynamic is supposed to be denoted by $f(x, t)=$ $\sin (10 \pi t) x_{2}(t)$ in the simulation. In order to satisfy Assumption $1, U$ is selected as $\operatorname{diag}\{0,1,0,0\}$. The initial value of state is taken to be $x(0)=[2,-2,3,0]$ and $\lambda$ is selected as 20 . Based on Theorem 6 , it can be solved that

$$
\begin{gathered}
L=\left[\begin{array}{llll}
0 & -0.0001 & 0.0019 & 0 \\
0 & -0.0001 & 0.0012 & 0
\end{array}\right], \\
K=\left[\begin{array}{llll}
0.2952 & 2.4053 & 0.3805 & 4.8333
\end{array}\right] .
\end{gathered}
$$

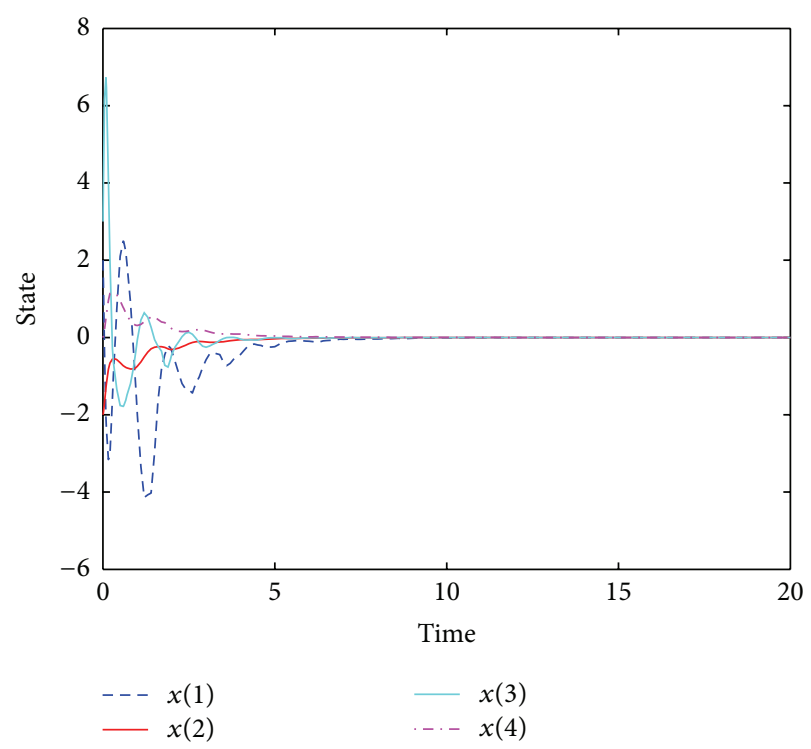

FIGURE 2: System performance using DOBC strategy for known nonlinearity.

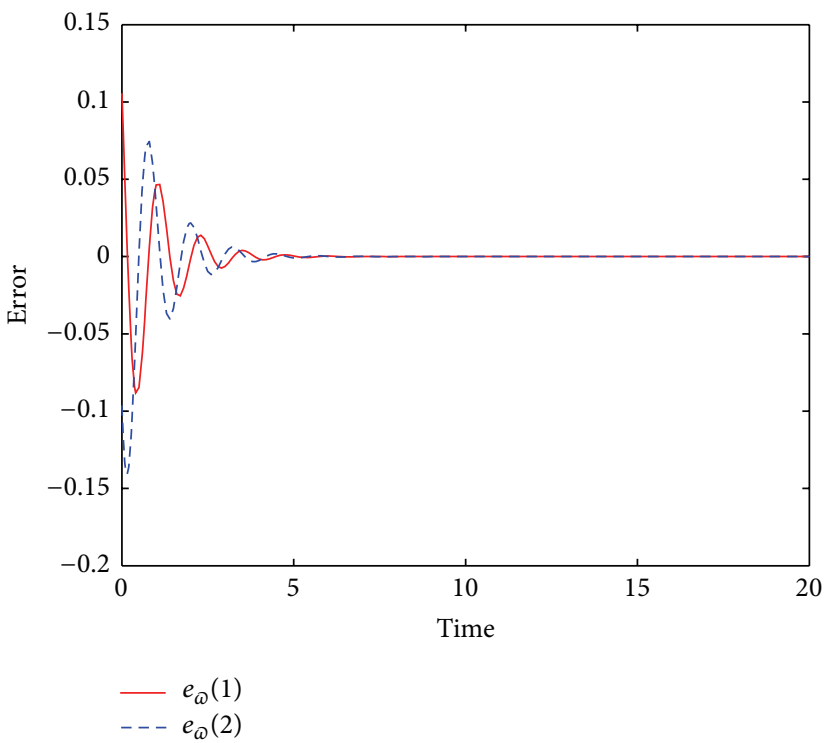

FIGURE 3: Estimation error of disturbance for known nonlinearity.

When the stochastic robust control strategy is applied to (33), which was first given in [13], it can be shown from Figure 1 that asymptotical stabilization in probability cannot be guaranteed in the presence of disturbance $v(t)$. Figures 2 and 3 show the system response and estimation error of system disturbance for the case with known nonlinearity, respectively. The simulation results show that asymptotical stabilization can be achieved using the method proposed in this paper and that the proposed disturbance observer is fine and effective.

Case 2 (with unknown nonlinearity). When nonlinear term $f(x, t)$ is unknown, we assume $f(x, t)=r(t) x_{2}(t)$ in 


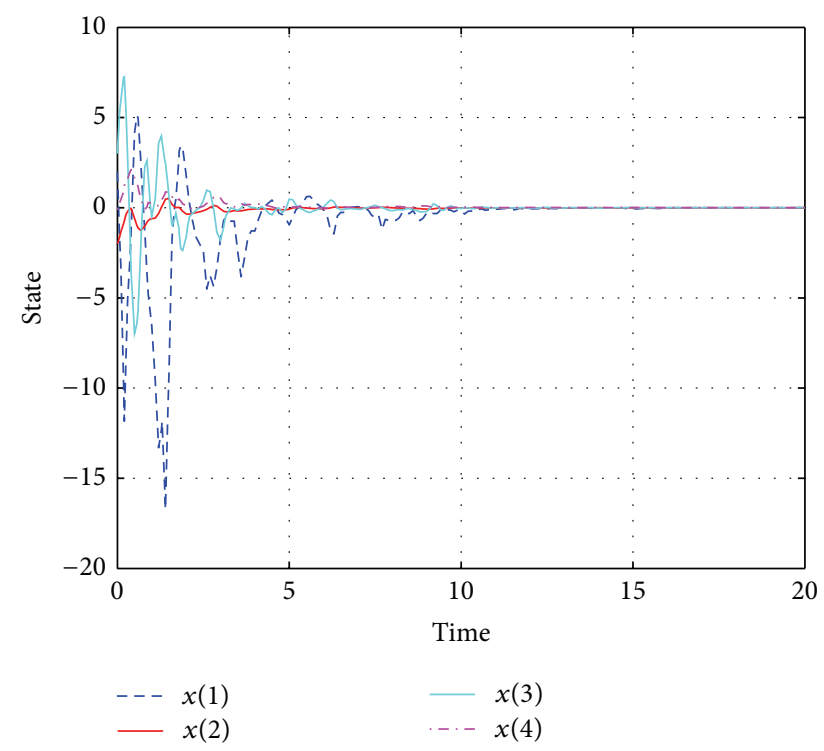

FIGURE 4: System performance using DOBC strategy for unknown nonlinearity.

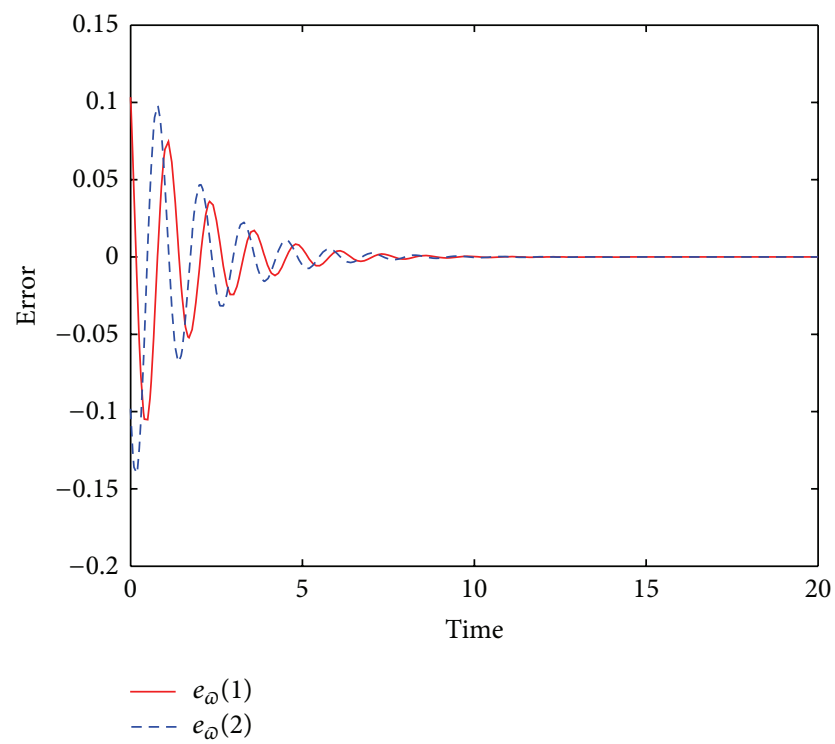

FIGURE 5: Estimation error of disturbance for unknown nonlinearity.

simulation, where $r(t)$ is supposed to be stochastic input that obeys uniform distribution. Based on Theorem 7, it can be solved that

$$
\begin{gathered}
L=\left[\begin{array}{llll}
0 & 0.0001 & 0.0011 & 0 \\
0 & 0.0001 & 0.0006 & 0
\end{array}\right], \\
K=\left[\begin{array}{llll}
0.2209 & 0.9695 & 0.2536 & 3.1946
\end{array}\right] .
\end{gathered}
$$

It is clear from Figure 4 that all states of system converge to zero and estimation errors of disturbance also converge to zero as shown in Figure 5. As has been shown above, we can see that asymptotical stabilization in probability is guaranteed and satisfied performance of the closed-loop control systems is achieved.

\section{Conclusion}

In this paper, the DOBC approach is investigated for a class of nonlinear systems with deterministic and stochastic disturbances. Feasible design procedures are proposed under different conditions to estimate and reject deterministic disturbance for the plants with known and unknown nonlinearity. Based on the estimation of disturbances, the composite control laws can guarantee the composite closed-loop systems to be global asymptotical stabilization in probability in the presence of disturbances. Simulation for an aircraft model shows the efficiency of the proposed algorithms.

\section{Acknowledgments}

This work was supported partly by the National Natural Science Foundation of China under Grants 61374131, 60925012, and 61121003 and the National Basic Research Program of China (973 Program) under Grant 2012CB720003.

\section{References}

[1] H. J. Kushner, Stochastic Stability and Control, Academic Press, New York, NY, USA, 1967.

[2] A. Isidori, Nonlinear Control Systems, Springer, New York, NY, USA, 3rd edition, 1995.

[3] E. D. Sontag, "A "universal" construction of Artstein's theorem on nonlinear stabilization," Systems \& Control Letters, vol. 13, no. 2, pp. 117-123, 1989.

[4] P. Florchinger, "A universal formula for the stabilization of control stochastic differential equations," Stochastic Analysis and Applications, vol. 11, no. 2, pp. 155-162, 1993.

[5] P. Florchinger, "Lyapunov-like techniques for stochastic stability," SIAM Journal on Control and Optimization, vol. 33, no. 4, pp. 1151-1169, 1995.

[6] P. Florchinger, "Feedback stabilization of affine in the control stochastic differential systems by the control Lyapunov function method," SIAM Journal on Control and Optimization, vol. 35, no. 2, pp. 500-511, 1997.

[7] P. Florchinger, "A passive system approach to feedback stabilization of nonlinear control stochastic systems," SIAM Journal on Control and Optimization, vol. 37, no. 6, pp. 1848-1864, 1999.

[8] Z. Pan and T. Başar, "Backstepping controller design for nonlinear stochastic systems under a risk-sensitive cost criterion," SIAM Journal on Control and Optimization, vol. 37, no. 3, pp. 957-995, 1999.

[9] H. Deng and M. Krstić, "Stochastic nonlinear stabilization. II. Inverse optimality," Systems \& Control Letters, vol. 32, no. 3, pp. 151-159, 1997.

[10] L. Wu, W. X. Zheng, and H. Gao, "Dissipativity based sliding mode control of switched stochastic systems," IEEE Transactions on Automatic Control, vol. 58, no. 3, pp. 785-793, 2013.

[11] Y. Y. Yin, Y. Shi, and F. Liu, "Constrained model predictive control on conven polyhedron stochastic linear parameter varying systems," International Journal of Innovative Computing, Information and Control, vol. 9, no. 10, pp. 4193-4204, 2013. 
[12] H. Q. Wang, B. Chen, and C. Lin, "Adaptive neural tracking control for a class of stochastic nonlinear systems with unknown dead-zone," International Journal of Innovative Computing, Information and Control, vol. 9, no. 8, pp. 3257-3269, 2013.

[13] D. Hinrichsen and A. J. Pritchard, "Stochastic $H^{\infty}$," SIAM Journal on Control and Optimization, vol. 36, no. 5, pp. 15041588, 1998.

[14] H. X. Li and P. P. J. van den Bosch, "A robust disturbance-based control and its application," International Journal of Control, vol. 58, no. 3, pp. 537-554, 1993.

[15] S. P. Chan, "Disturbance observer for robot manipulators with application to electronic components assembly," IEEE Transactions on Industrial Electronics, vol. 42, no. 5, pp. 487493, 1995.

[16] R. Bickel and M. Tomizuka, "Passivity-based versus disturbance observer based robot control: equivalence and stability," Journal of Dynamic Systems, Measurement and Control, vol. 121, no. 1, pp. 41-47, 1999.

[17] Y. Oh and W. K. Chung, "Disturbance-observer-based motion control of redundant manipulators using inertially decoupled dynamics," IEEE/ASME Transactions on Mechatronics, vol. 4, no. 2, pp. 133-146, 1999.

[18] Y. Huang and W. Messner, "A novel disturbance observer design for magnetic hard drive servo system with a rotary actuator," IEEE Transactions on Magnetics, vol. 34, no. 4, pp. 1892-1894, 1998.

[19] C. J. Kempf and S. Kobayashi, "Disturbance observer and feedforward design for a high-speed direct-drive positioning table," IEEE Transactions on Control Systems Technology, vol. 7, no. 5, pp. 513-526, 1999.

[20] J. Ishikawa and M. Tomizuka, "Pivot friction compensation using an accelerometer and a disturbance observer for hard disk drives," IEEE/ASME Transactions on Mechatronics, vol. 3, no. 3, pp. 194-201, 1998.

[21] L. Guo and W.-H. Chen, "Disturbance attenuation and rejection for systems with nonlinearity via DOBC approach," International Journal of Robust and Nonlinear Control, vol. 15, no. 3, pp. 109-125, 2005.

[22] W. Chen, "Disturbance observer based control for nonlinear systems," IEEE/ASME Transactions on Mechatronics, vol. 9, no. 4, pp. 706-710, 2004.

[23] L. Wu, X. Su, and P. Shi, "Output feedback control of markovian jump repeated scalar nonlinear systems," IEEE Transactions on Automatic Control, 2013.

[24] X. Yao and L. Guo, "Composite anti-disturbance control for Markovian jump nonlinear systems via disturbance observer," Automatica, vol. 49, no. 8, pp. 2538-2545, 2013.

[25] L. Xie, X. He, W. Zhang, and X. Xu, "Robust $H_{\infty}$ control for nonlinear uncertain stochastic systems with multiple time delays," Control and Decision, vol. 16, pp. 779-782, 2001.

[26] S. Tong, L. Wu, X. Wei, and L. Guo, "Disturbance-observerbased control approach for a class of nonlinear systems with neutral uncertainty," in Proceedings of the Chinese Control and Decision Conference, pp. 4717-4721, Yantai, China, July 2008.

[27] X. Wei and L. Guo, "Composite disturbance-observer-based control and $H_{\infty}$ control for complex continuous models," International Journal of Robust and Nonlinear Control, vol. 20, no. 1, pp. 106-118, 2010.

[28] L. Guo, X. Y. Wen, and X. Xin, "Hierarchical composite anti-disturbance control for robotic systems using robust disturbance observer," in Proceedings of the Robot Intelligence, Advanced Information and Knowledge, pp. 229-243, 2010.
[29] P. E. Caines and K. Nassiri-Toussi, "On the adaptive stabilization and ergodic behaviour of stochastic systems with jump-Markov parameters via nonlinear filtering," in Topics in Stochastic Systems: Modelling, Estimation and Adaptive Control, vol. 161 of Lecture Notes in Control and Information Science, pp. 185-215, Springer, Berlin, Germany, 1991.

[30] K. Nassiri-Toussi and P. E. Caines, "On the adaptive stabilization and ergodic behaviour of stochastic jump parameter systems via non-linear filtering," in Proceedings of the 30th IEEE/CDC Conference on Decision and Control, December 1991.

[31] I. R. Petersen and C. V. Hollot, "A Riccati equation approach to the stabilization of uncertain linear systems," Automatica, vol. 22, no. 4, pp. 397-411, 1986. 


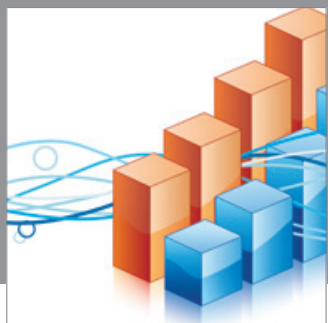

Advances in

Operations Research

mansans

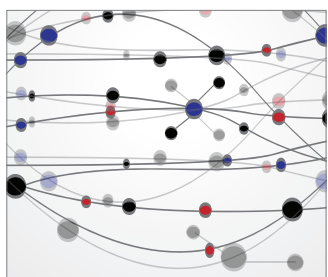

The Scientific World Journal
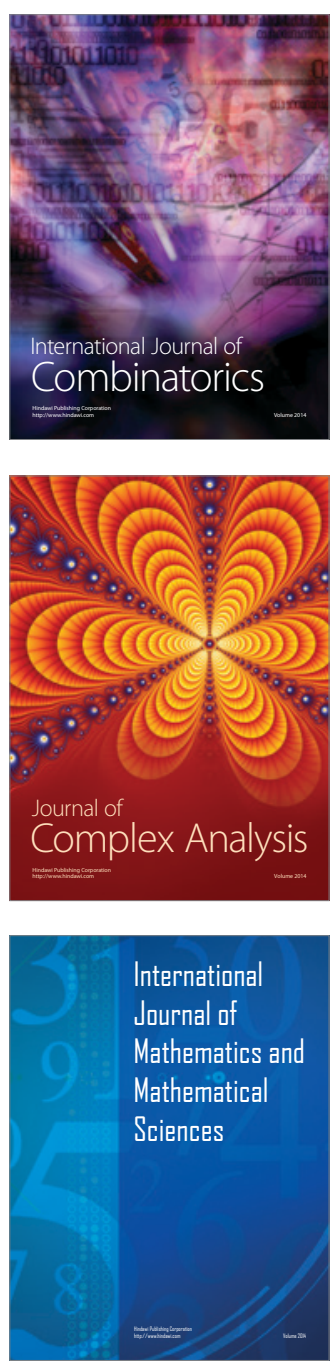
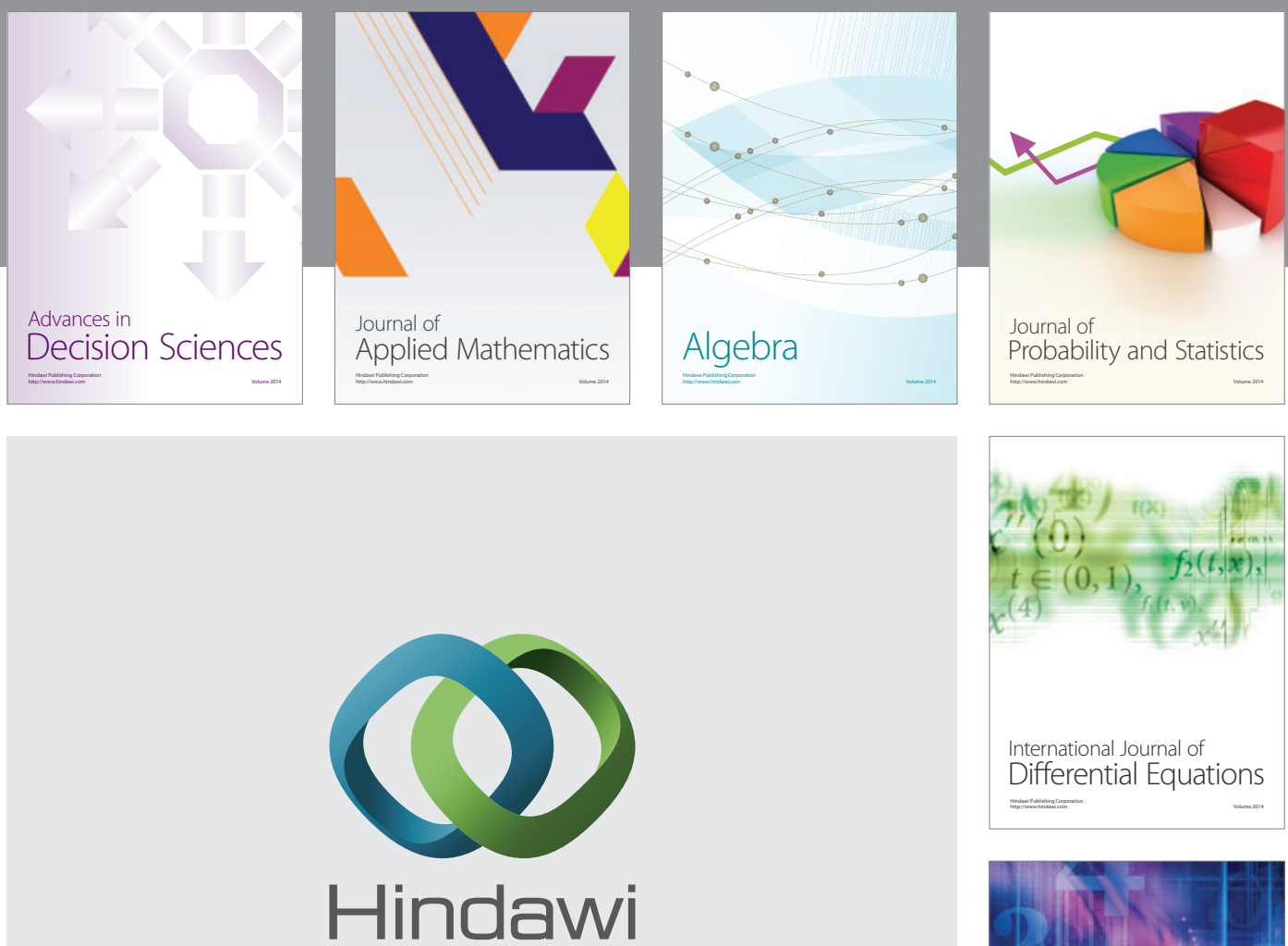

Submit your manuscripts at http://www.hindawi.com
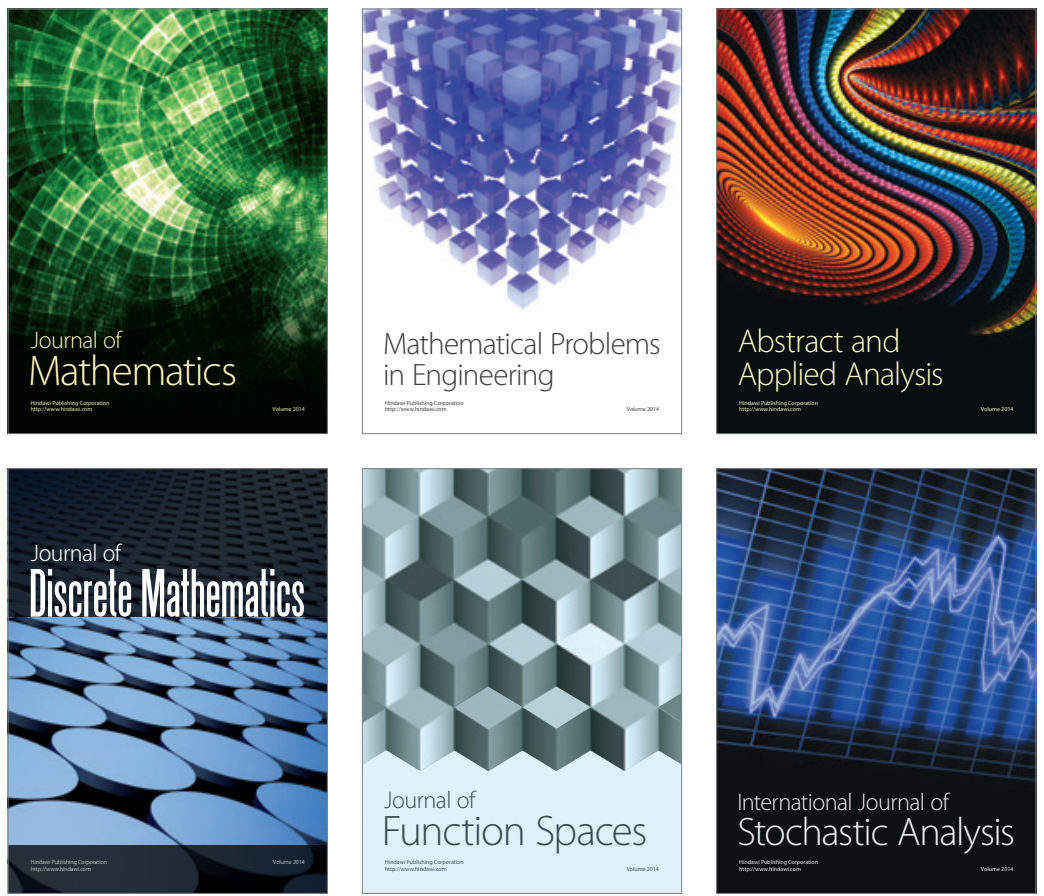

Journal of

Function Spaces

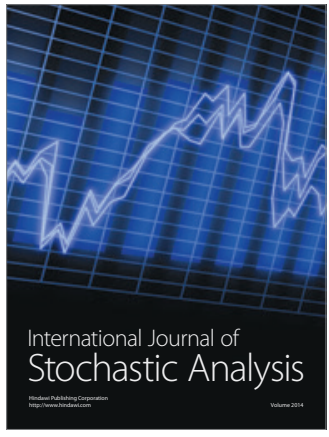

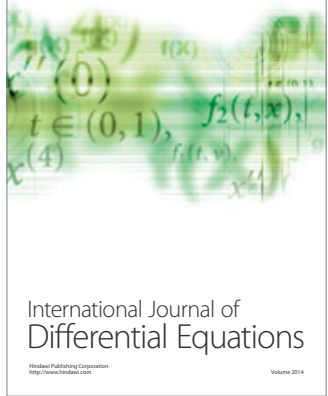
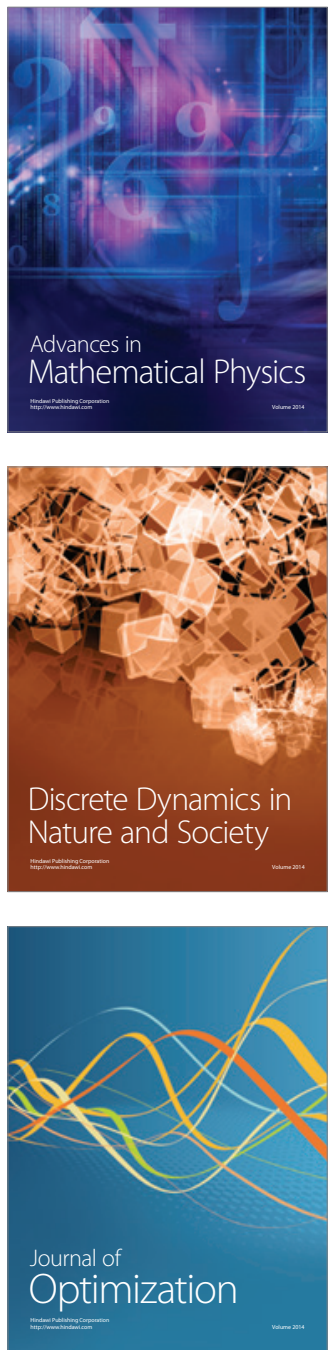